\title{
Ribosomal DNA evolution and gene conversion in Nicotiana rustica
}

\author{
R Matyasek ${ }^{1}$, KY Lim², A Kovarik ${ }^{1}$ and AR Leitch ${ }^{2}$ \\ ${ }^{1}$ Institute of Biophysics, Academy of Sciences of the Czech Republic, CZ-61265 Brno, Czech Republic; ${ }^{2}$ School of Biological Sciences, \\ Queen Mary, University of London, London E1 4NS, UK
}

\begin{abstract}
Genomic in situ hybridisation was used to confirm that Nicotiana rustica $(2 n=4 x=48)$ is an allotetraploid between $N$. paniculata $(2 n=2 x=24$, maternal $P$-genome donor) and $N$. undulata $(2 n=2 x=24$, paternal U-genome donor), their progenitors or species closely related to them. Fluorescent in situ hybridisation showed that $N$. paniculata has one $5 S$ and two 18-5.8-26S rDNA loci whereas $N$. undulata has an additional 18-5.8-26S rDNA locus. $N$. rustica has the sum of the loci found in these putative parents. The sizes of the 185.8-26S rDNA loci indicate that the number of rDNA units on the U-genome chromosomes has amplified; perhaps this is associated with a concomitant reduction in the number of units on P-genome chromosomes. Restriction fragment length polymorphism analysis of the intergenic spacer
\end{abstract}

(IGS) of the 18-5.8-26S rDNA units in N. rustica and the two progenitor diploids revealed that about $80 \%$ of IGS sequences in N. rustica are of an N. undulata type and $20 \%$ of $N$. paniculata type. These data indicate that interlocus sequence homogenisation has caused the replacement of many $N$. paniculata-type IGSs in $N$. rustica with an $N$. undulata-type of sequence. It is probable that subsequent to this replacement there has been sequence divergence at the $5^{\prime}$ end of the IGS. As in tobacco, an allotetraploid between $N$. sylvestris and $N$. tomentosiformis, the direction of the IGS interlocus conversion is towards the paternal genome donor.

Heredity (2003) 91, 268-275. doi:10.1038/sj.hdy.6800333

Keywords: allopolyploid; rDNA; gene conversion; interphase; concerted evolution

\section{Introduction}

Polyploidy is a major force in plant evolution, with as many as $80 \%$ of angiosperm species being polyploid (Leitch and Bennett, 1997). Furthermore, DNA sequencing programmes are revealing that many apparently diploid species are palaeopolyploid (Soltis and Soltis, 1999). Plant evolution appears to have involved a series of genome duplication events followed by selection for genome diploidisation. Many genetic events are associated with polyploidy, including chromosomal translocations, gene conversion, as well as loss, gain, amplification and reduction of sequences. However, the universality of these genetic changes, the factors that govern their extent, direction and occurrence are not well understood. How the events attributed to polyploidy differ from events that occur during diploid speciation is the subject of controversy.

One of the best characterised genetic events associated with allopolyploidy is gene conversion at the 18-5.8-26S nuclear ribosomal DNA (rDNA) loci in Nicotiana tabacum L. (Kovarik et al, 1996; Volkov et al, 1999; Lim et al, 2000a). N. tabacum (tobacco) is an allotetraploid $(2 n=4 x=48)$ that formed within the last 6 million years (Olmstead

Correspondence: AR Leitch, School of Biological Sciences, Queen Mary University of London, London E1 4NS, UK

E-mail: A.R.Leitch@qmul.ac.uk

Received 24 July 2002; revised 26 February 2003; accepted 14 April 2003 and Palmer, 1991) between N. sylvestris Speg. \& Comes $(2 n=2 x=24)$, the maternal $S$ genome donor, and $N$. tomentosiformis L. $(2 n=2 x=24)$, the paternal T genome donor (Goodspeed, 1954; Chase et al, 2002; Murad et al, 2002). N. sylvestris has three rDNA loci, whereas $N$. tomentosiformis has one locus; the sum of these is found in tobacco (Parokonny and Kenton, 1995; Lim et al, 2000a). However, an analysis of the rDNA units shows that over $90 \%$ of the units are of an $N$. tomentosiformis type whereas the remainder are of an $N$. sylvestris type (Lim et al, 2000b). Thus, these data indicate that gene conversion has homogenised most, but not all units.

In this paper we investigate evolutionary processes that have influenced the structure of the 18-5.8-26S rDNA loci in N. rustica L. $(2 n=4 x=48)$, a putative allotetraploid between progenitors of the diploid species $N$. paniculata L. $(2 n=2 x=24)$, and N. undulata Ruiz and Pavlon $(2 n=4 x=48)$. Phylogenetic studies of the chloroplast sequence suggest that the ancestor of $N$. paniculata and/or N. knightiana (section Paniculatae) may be the maternal genome donor of N. rustica (Aoki and Ito, 2000; Chase et al, 2003). The closest relative of $N$. undulata (section Undulatae) is N. glutinosa although Goodspeed (1954) placed N. glutinosa in section Tomentosae. It was Goodspeed's (1954) analyses of morphological data, breeding experiments and karyotypes that led him to the view that $N$. paniculata and $N$. undulata were most closely related to the diploid parents of $N$. rustica, and for this reason these species were analysed here. We used 
genomic in situ hybridisation (GISH) to verify that $N$. rustica is indeed an allopolyploid of these species or a close relative of them. We compared patterns of rDNA evolution in N. rustica with $N$. tabacum. We investigated whether the number and location of the rDNA loci is the sum of that found in the putative parents, and whether concerted evolution has altered or homogenised units as has occurred in N. tabacum. The results are also interpreted in the light of other rDNA unit arrays previously described in allopolyploids in Triticum (Flavell, 1990), Brassica (Chen and Pikaard, 1997) and Gossypium (Wendel et al, 1995a).

\section{Materials and methods}

\section{Plant material and chromosome preparations}

Root tips of N. paniculata L, N. rustica L. and N. undulata Ruiz \& Pavlon (all from USDA, Agricultural Research Station, North Carolina State University, USA) were used in this study. For fluorescent in situ hybridisation (FISH), root tips from pot-grown plants were analysed without pretreatment, or following pretreatment with a saturated aqueous solution of Gammexane ${ }^{\mathbb{R}}$ (hexachlorocyclohexane, Sigma Aldrich) for $4 \mathrm{~h}$. All root tips were fixed in 3:1 absolute ethanol:glacial acetic acid for more than a week. Chromosome squashes were prepared following enzyme softening of material as described by Leitch et al (2001).

\section{Restriction endonuclease digestion and Southern hybridisation}

Genomic DNA was extracted from young leaves of these plants using the method of Saghai-Maroof et al (1984) with modifications according to Kovarik et al (1996). Methods followed those by Sambrook et al (1989). DNAs was digested to completion with excess of restriction endonucleases and fractionated in 1\% agarose by gel electrophoresis. DNA was transferred to a Hybond $\mathrm{N}+$ membrane (Amersham Pharamacia Biotech.). Southern hybridisation was carried out under high-stringency conditions (Fulnecek et al, 2002) using a heat-denatured ${ }^{32} \mathrm{P}$-labelled rDNA probe that was either (i) a $1.7 \mathrm{~kb}$ EcoRI fragment of the 18S rRNA gene subunit from Solanum lycopersicum L. (tomato, Kiss et al, 1989b, accession number X51576) or (ii) a $220 \mathrm{bp}$ fragment of the $26 \mathrm{~S}$ rRNA gene subunit from S. lycopersicum (Kiss et al, 1989a, accession number X13557) obtained by PCR amplification of the region between +2901 (5'-GAA TTC ACC CAA GTG TTG GGA T-3') and +3121 (5'-AGA GGC GTT CAG TCA TAA TC-3') with respect to the start of the $26 \mathrm{~S}$ rDNA subunit (Kiss et al, 1989a). Labelling of DNA probes was carried out by a random primed method using ${ }^{32} \mathrm{P}-\mathrm{dCTP}$ (Dekaprime kit, Ambion ${ }^{\mathrm{TM}}$, USA).

\section{Probes for in situ hybridisation}

(1) N. undulata total genomic DNA was labelled by nick translation with digoxigenin-11-dUTP (Roche Biochemicals) and N. paniculata total genomic DNA with biotin-16-dUTP (Sigma Aldrich) following Leitch et al (2001).

(2) The entire plasmid containing pTa71 (isolated by Gerlach and Bedbrook, 1979), which includes the 185.8-26S rDNA subunits and the intergenic spacer
(IGS) isolated from Triticum aestivum, was labelled with digoxigenin-11-dUTP or biotin-16-dUTP by nick translation.

(3) pTZ19-R: the 5S rDNA unit isolated from N. rustica (Venkateswarlu et al, 1991) was labelled with digoxigenin-11-dUTP or biotin-16-dUTP by nick translation.

Fluorescent in situ hybridisation (FISH): FISH was carried out as described by Leitch et al (1994), with modifications as described by Lim et al (1998). Briefly, slides were pretreated with $100 \mu \mathrm{g} / \mathrm{ml}$ RNase A for $1 \mathrm{~h}$ and $0.25 \mu \mathrm{g} / \mathrm{ml}$ pepsin for $5 \mathrm{~min}$ followed by denaturation in $70 \%$ formamide in $2 \times$ SSC $(0.3 \mathrm{M}$ sodium chloride, $0.03 \mathrm{M}$ sodium citrate) at $70^{\circ} \mathrm{C}$ for $2 \mathrm{~min}$. The hybridisation mix contained $50 \%(\mathrm{v} / \mathrm{v})$ formamide, $10 \%(\mathrm{w} / \mathrm{v})$ dextran sulphate and $0.1 \%(\mathrm{w} / \mathrm{v})$ sodium dodecyl sulphate in $2 \times$ SSC. For GISH, the hybridisation mixture contained $8 \mu \mathrm{g} / \mathrm{ml}$ digoxigeninlabelled $N$. undulata DNA and $8 \mu \mathrm{g} / \mathrm{ml}$ biotin-labelled $N$. paniculata DNA. A concentration of $4 \mu \mathrm{g} / \mathrm{ml}$ DNA was used in the mixture when using the cloned probes pTa71 and pTZ19-R. After overnight hybridisation at $37^{\circ} \mathrm{C}$, the slides were washed in $20 \%(\mathrm{v} / \mathrm{v})$ formamide in $0.1 \times$ SSC at $42{ }^{\circ} \mathrm{C}$ at an estimated hybridisation stringency of 80 $85 \%$. Sites of probe hybridisation were detected using $20 \mu \mathrm{g} / \mathrm{ml}$ fluorescein conjugated antidigoxigenin IgG (Roche Biochemicals) and $5 \mu \mathrm{g} / \mathrm{ml}$ Cy3 conjugated avidin (Amersham Biosciences). Chromosomes were counterstained with $2 \mu \mathrm{g} / \mathrm{ml}$ DAPI $\left(4^{\prime}, 6\right.$-diamidino-2phenylindole) in $4 \times$ SSC, mounted in Vectashield medium (Vector Laboratories), examined using a Leica DMRA2 epifluorescent microscope and photographed with an Orca ER camera. Images were processed for colour balance, contrast and brightness uniformly.

\section{Results}

In situ hybridisation showing the distribution of ribosomal DNA

Using FISH, it was shown that $N$. paniculata has two loci (four sites) of 18-5.8-26S rDNA at the distal end of the short arm of two metacentric chromosomes (Figure 1a). One of these loci was larger than the other, enabling the homologues to be distinguished at metaphase (Figure 1a) and interphase (Figure 2a). Double labelling with 5S rDNA reveals a single locus near the centromere of an additional metacentric chromosome (Figure 1a). In contrast, FISH to N. undulata reveals three loci of 185.8-26S rDNA at the distal end of the short arm of three metacentric chromosomes, and one of these chromosomes carries a more proximal 5S rDNA locus (Figure 1b). N. rustica has the sum of the loci observed in these putative parents (Figure 1c).

GISH can be used to distinguish the genomic origin of the chromosomes in allopolyploids (Heslop-Harrison, 1992; Gill and Friebe, 1998) and has been used to confirm the ancestry of allopolyploids in a number of species including tobacco (Parokonny and Kenton, 1995; Lim et al., 2000a). Figure $1 \mathrm{~d}$ shows that the method distinguishes between chromosomes inherited from progenitors of $N$. undulata and N. paniculata, the U- and P-genomes of $N$. rustica respectively. Even though the positions and numbers of the rDNA loci in N. rustica 
270
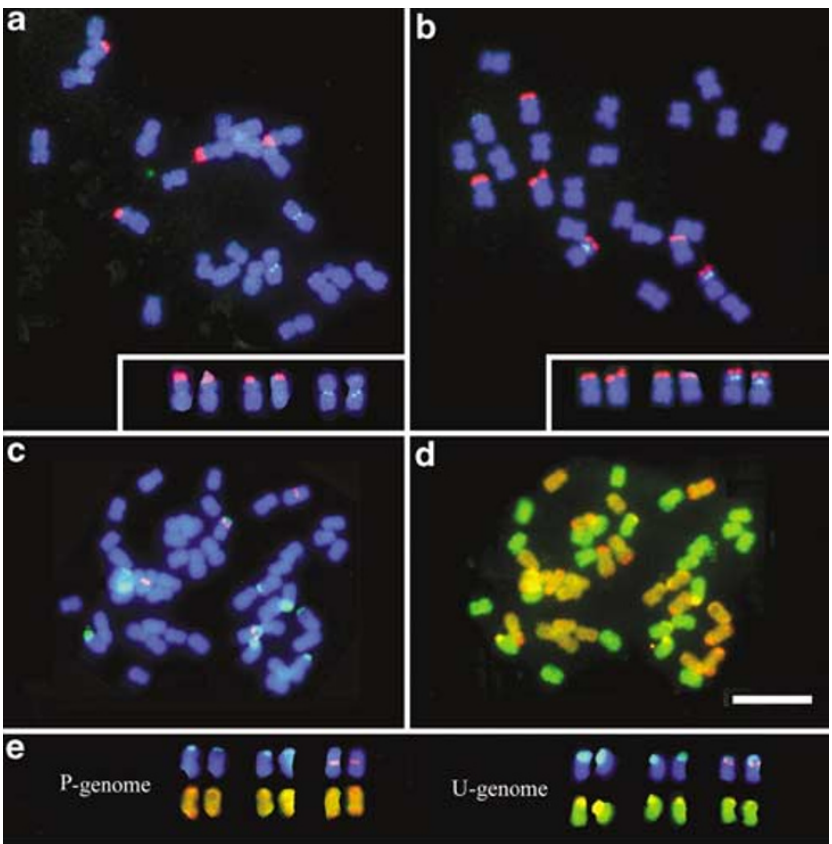

Figure 1 FISH to metaphase spreads of $N$. paniculata (a) and $N$ undulata (b) probed with biotin-labelled pTa 71 (pink) and digoxigenin-labelled pTZ19-R (green) to reveal sites of 18-5.8-26S rDNA and 5S rDNA loci, respectively. (c) Metaphase of N. rustica probed with digoxigenin-labelled pTa 71 and biotin-labelled pTZ19$R$. The counterstain is DAPI (blue) for DNA. In (d), the metaphase shown in (c) was reprobed by GISH using total genomic DNA from $N$. undulata (green) and N. paniculata (orange). This enables the genomic origin of the chromosomes to be determined (e). Those chromosomes in (c) and (d) that carry rDNA have been given a genome identity. Scale bar $10 \mu \mathrm{m}$.

occur as the sum of the organisation found in the parents (Figure $1 \mathrm{a}$ and $\mathrm{b}$ ), there are some notable differences. The two largest 18-5.8-26S rDNA loci in N. rustica occur on U-genome chromosomes and can be distinguished from each other by size (Figure 1c-e), and the P-genome 185.8-26S rDNA loci are small compared with N. paniculata. Assuming that the rDNA signal distribution reflects that found in the true parents of $N$. rustica, the data indicate copy number expansion at one or two U-genome loci, perhaps with a concomitant reduction at P-genome loci.

Many hundreds of meristematic, circular interphase nuclei were examined from several gammexane-treated root tips of the three species analysed. The two 18-5.8-26S rDNA loci in N. paniculata are typically expressed at interphase, with the proximal end of the locus being decondensed and the distal end remaining condensed and heterochromatic (Figure 2a and b). Likewise in $N$. undulata, the three 18-5.8-26S rDNA loci are decondensed in a similar manner (Figure $2 \mathrm{c}$ and $\mathrm{d}$ ). In interphases of $N$. rustica, up to four (often two) of the 10 sites are condensed and unassociated with nucleoli (two sites like this are indicated in Figure 2e). The two largest 18-5.8$26 \mathrm{~S}$ rDNA loci in N. rustica, carried on U-genome chromosomes, show interlocus variability in the level of chromatin decondensation, but with most condensed rDNA occurring at the distal end of the locus (Figure 2e and $\mathrm{f}$ ). The third rDNA locus on a U-genome chromosome is not materially larger than the P-genome loci, and the sites are not readily distinguishable at interphase. But at metaphase, the third U-genome chromosome carrying 18-5.8-26S rDNA can be identified by the linked 5S rDNA locus. In these cells this U-genome chromosome can be seen to carry a secondary constriction suggesting locus activity at the preceding interphase (Figure $2 \mathrm{~g}$ and $\mathrm{h}$, arrow). An examination of N. rustica root-tip cells without pretreatment shows that the decondensed rDNA is more dispersed, but the profile of locus decondensation remained similar with silent sites unassociated with nucleoli apparent at interphase (Figure $2 \mathrm{i}-1$ ). Thus, the rDNA loci of $N$. rustica show variable activity both between and within loci as reported for other plant species (Vieira et al, 1990) and that the least active sites appear to occur on P-genome chromosomes.

Genetic structure of the IGS in N. rustica and its putative parents

The genetic structure of the IGS of the 18-5.8-26S rDNA gene unit in N. rustica and its putative diploid parents $N$. paniculata and $N$. undulata was analysed using Southern hybridisation. Figure $3 a$ shows Hind IIIdigested DNA of N. paniculata, N. rustica and N. undulata probed with the $26 \mathrm{~S}$ rDNA probe. The restriction profile shows a band of $9.5 \mathrm{~kb}$ in $N$. paniculata and slightly greater than $10 \mathrm{~kb}$ in N. undulata. The same bands are revealed with the $18 \mathrm{~S}$ rDNA probe (data not shown), providing evidence that these restriction fragments are monomeric units of the 18-5.8-26S rDNA transcribed genic region and the IGS. A similar-sized 18-5.8-26S rDNA unit is found in related Nicotiana species (Kovarik et al, 1996; Volkov et al, 2001). However, N. rustica does not have the sum of the bands seen in N. paniculata and N. undulata. Instead a more complex profile is observed with several bands occurring between 9.5 and $10.5 \mathrm{~kb}$, and only some of these are directly coincident with those seen in the diploids.

A range of restriction enzymes was used to analyse size heterogeneity in the 18-5.8-26S rDNA unit of $N$. rustica and to investigate any polymorphisms in the IGS of all three species. When there are two or more restriction sites in the rDNA unit, and one or more are in the IGS, then the $18 \mathrm{~S}$ rDNA probe identifies polymorphisms at the $3^{\prime}$ end of the IGS. Digestion with SspI and DraI restriction enzymes and probing with $18 \mathrm{~S}$ rDNA generates fragments that show little polymorphism between species (Figure $3 b$ ). In contrast when genomic DNA is restricted with EcoRV a distinct species-specific hybridisation profile is observed, allowing the two diploid species to be distinguished. With EcoRV it is evident that only a small fraction (ca 20\%) of the rDNA restriction fragments in N. rustica appear to be similar to those in N. paniculata (Figure 3b arrow) - (P-type units in N. rustica), - whereas the majority of IGS copies have a restriction profile that closely resembles $N$. undulata, U-type units.

Reprobing the membrane containing $S s p 1, D r a I$ and EcoRV digested DNA (Figure 3b) with the 26S rDNA probe (Figure 3c) reveals IGS polymorphism at the $5^{\prime}$ end of the sequence. In EcoRV digested DNA, a small fraction of the bands in N. rustica are shared with N. paniculata and N. undulata, whereas in SspI and DraI restricted DNA only some bands are shared with N. undulata. Most of the hybridisation bands in N. rustica are unique, indicating considerable genetic change at the $5^{\prime}$ end of 

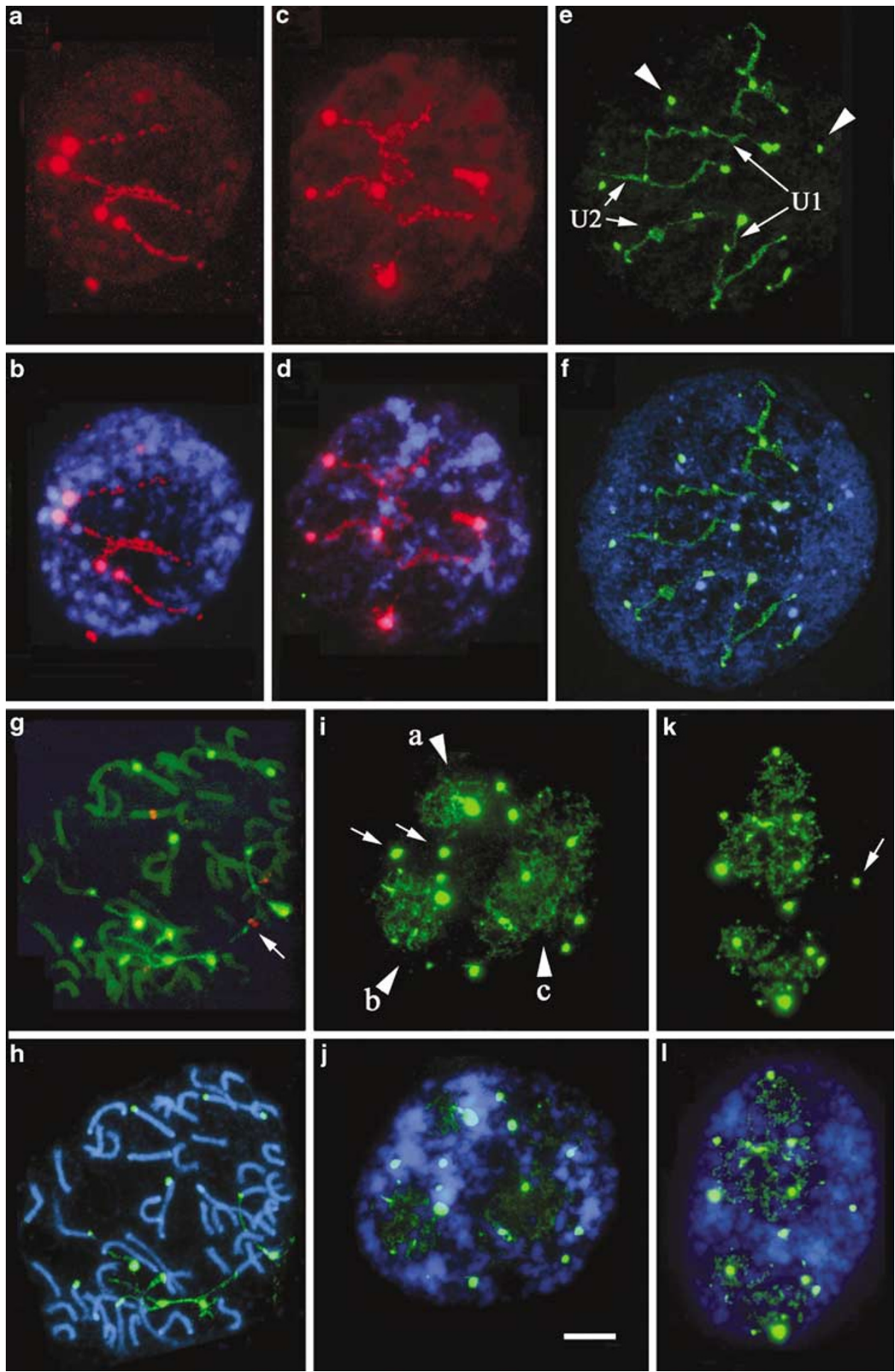

Figure 2 Interphases of $N$. paniculata $(\mathbf{a}, \mathbf{b}), N$. undulata $(\mathbf{c}, \mathbf{d})$ and $N$. rustica $(\mathbf{e}, \mathbf{f})$ probed with pTa71 labelled either with digoxigenin-FITC (yellow/green) or biotin-avidin Cy3 (orange/red). The counterstain for DNA is DAPI (blue). (a-h). Cells from gammexane-treated roots. (a) Two pairs of different-sized 18-5.8-26S rDNA loci and differential decondensation withinin each locus are shown. The red dot outside the nucleus is background. (c,d) The three pairs of rDNA loci show signs of decondensation at interphase. The arrow heads in (e) show small rDNA sites that are condensed, inactive and unassociated with nucleoli. The arrows point to the two largest rDNA loci from U-genome chromosomes. Each of these sites show variable condensation. (g) Pro-metaphase of N. rustica probed with digoxigenin-labelled pTa71 (185.8-26S rDNA, green) and biotin-labelled pTZ19-R (5S rDNA, red) rDNA. The arrow points to a U-genome chromosome carrying both $5 \mathrm{~S}$ and 18-5.8-26S rDNA loci, the latter having a secondary constriction. (i-1) Interphases from untreated root tips (without gammexane). The nucleus $(\mathbf{i}, \mathbf{j})$ has three nucleoli $(\mathrm{a}-\mathrm{c})$ containing dispersed, probably active rDNA and peripheral blocks of condensed rDNA. There are at least one $(\mathbf{k}, \mathbf{l})$ or two $(\mathbf{i}, \mathbf{j})$ rDNA sites (arrows) that are condensed, heterochromatic and unassociated with nucleoli. The scale bar represents $13 \mu \mathrm{m}$ $(\mathbf{a}-\mathbf{d}), 10 \mu \mathrm{m}(\mathbf{e}-\mathbf{h})$, and $12 \mu \mathrm{m}(\mathbf{e}-\mathbf{h})$. 
a

b

26S rDNA

HindIII

(kb) $\mathrm{m} 2 \mathrm{P}$ R U

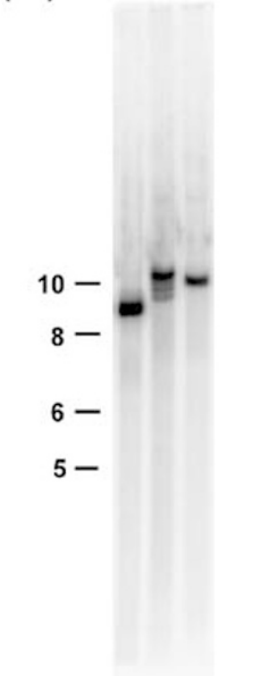

\section{$18 S$ rDNA}

Eco RV Ssp I Dra I

(kb) m1 PRU PRU PRU m2 (kb) c

\section{S rDNA}

EcoRV Ssp I Dra I

(kb) m1 PRU PR U PRU m2 (kb) d

\section{$26 \mathrm{~S}$ rDNA}

BstNI

P R U m1 (kb)
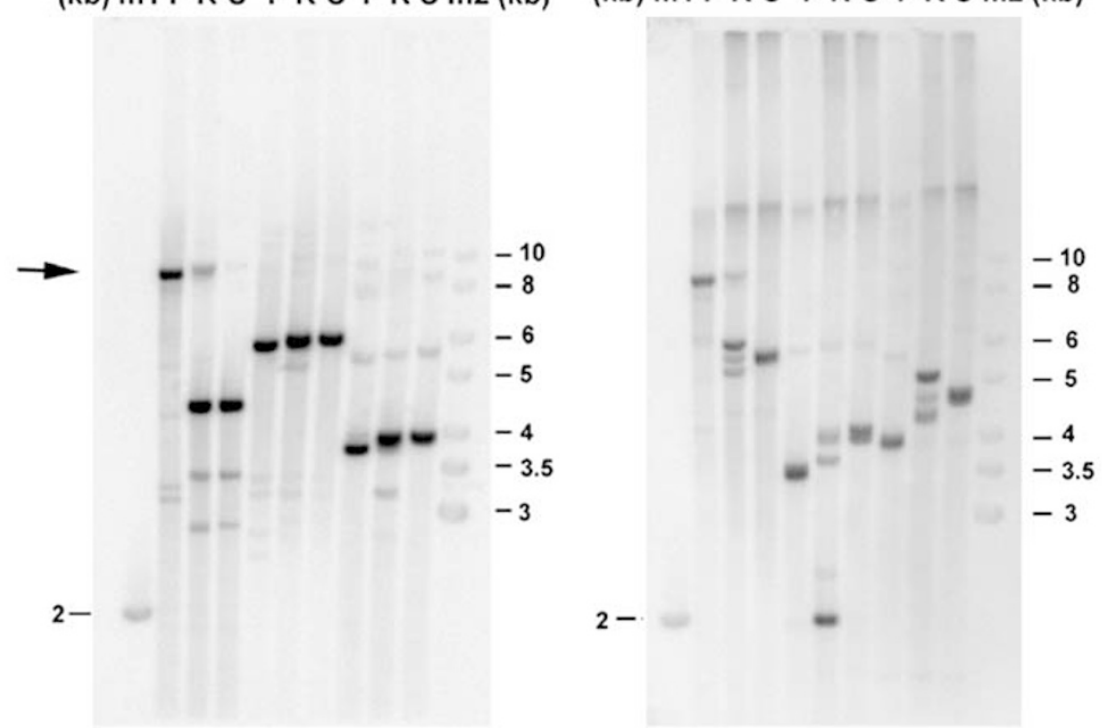

Figure 3 Southern hybridisation showing the overall structure of 18-5.8-26S rDNA units in N. paniculata (P), N. rustica (R) and N. undulata (U). The genomic DNA was cut with restriction enzymes and probed with $26 \mathrm{~S}(\mathbf{a}, \mathbf{c}, \mathbf{d})$ and $18 \mathrm{~S}(\mathbf{b}) \mathrm{rDNA}$ as indicated. The arrow in $\mathbf{b}$ indicates those units in N. rustica that are similar to units found in N. paniculata. m1: 100 bp DNA ladder (Gibco BRL); m2: 1 kb ladder (MBI Fermentas).

the IGS (towards the 26S rRNA gene) in the vast majority of N. rustica units. Nevertheless, an N. undulata-like character of $N$. rustica units can be observed in BstN1 restricted DNA probed with $26 \mathrm{~S}$ rDNA (Figure 3d). Reprobing the BstNI-digested genomic DNA with the 5S rDNA probe revealed that N. rustica has a pattern that is additive of that seen in the parents (data not shown).

Methylation patterns in $N$. rustica and its putative parents Digestion with EcoRV and probing with $18 \mathrm{~S}$ or $26 \mathrm{~S}$ rDNA probes produced species-specific restriction patterns that can be exploited to determine levels of DNA methylation in parental rDNA gene families in N. rustica. Double digests of genomic DNAs were carried out using EcoRV and the cytosine methylation-sensitive enzymes Fnu4HI, XhoI and PstI (Figure 4). XhoI is sensitive to methylation at CTmCGAG and has a conserved target site within the 26S gene of most plant species (as determined from plant DNA sequence databases). When $N$. rustica is digested with methylation-insensitive EcoRV, a small proportion of units appear like $N$. paniculata as shown previously (Figure $3 \mathrm{~b}$ ). These units migrating as an $\sim 9 \mathrm{~kb}$ EcoRV band are unaffected by Xho I digestion (Figure 4) in $N$. rustica, indicating that they are heavily methylated. However, in N. paniculata a significant fraction of this $9 \mathrm{~kb} E c o R V$ band is digested by XhoI into $5.5 \mathrm{~kb}$ (strong) and $7.4 \mathrm{~kb}$ (weak) bands. It is this undermethylated fraction of rDNA units that is missing in N. rustica. Using Fnu4HI (sensitive to methylation at GmCNGC) in the double digest with EcoRV, and probing with $18 \mathrm{~S}$ rDNA generates a complex array of bands (Figure 4). Once again many of the bands in N. rustica are similar to bands in N. undulata and absent in N. paniculata. The enzymes XhoI, PstI (sensitive to methylation at mCTGmCAG) and a

b

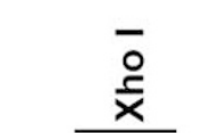

(kb) m PRU

(kb) m PRU

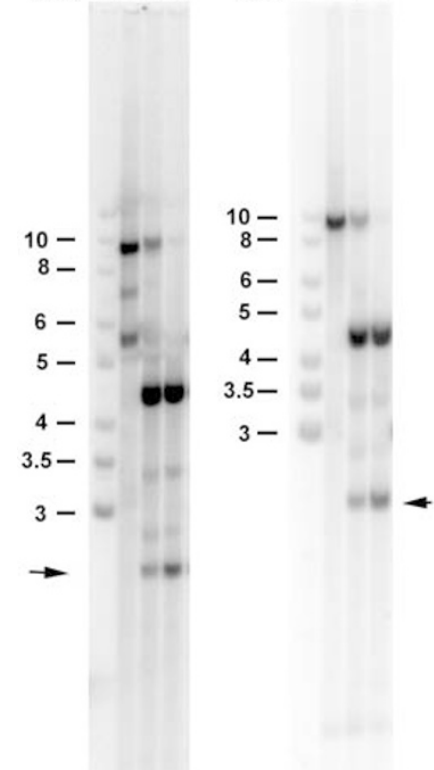

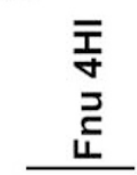

(kb) $\mathrm{MPRU}$

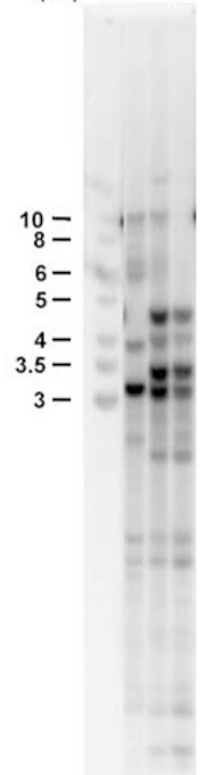

Figure 4 Southern hybridisation to determine methylation levels of 18-5.8-26S rDNA units in $N$. paniculata (P), N. rustica (R) and N. undulata (U). The genomic DNA was digested with EcoRV and subsequently with methylation-sensitive enzymes as indicated. The blots were probed with $18 \mathrm{~S}$ rDNA. The arrow shows short bands that are more prominent relative to the longer bands in N. undulata than is observed in $N$. rustica. m: $1 \mathrm{~kb}$ DNA ladder. 
Fnu4HI also reveal subtle differences in methylation levels between $N$. undulata and $N$. rustica, with a larger fraction of smaller fragment sizes in $N$. undulata, indicating slightly less cytosine methylation compared with N. rustica.

\section{Discussion}

The numbers and locations of 18-5.8-26S and 5S rDNA loci in $N$. rustica and the strong GISH signal strongly support the hypothesis that $N$. rustica is an allopolyploid between $N$. paniculata and $N$. undulata, their progenitors, or closely related species. It is apparent that since allopolyploidy there has been little change in the overall structure of the chromosomes. Furthermore, if there was substantial intermixing of the genomes subsequent to allopolyploid formation, through, for example, retrotranspostion or sequence homogenisation, it would be unlikely that GISH would work effectively. The fact that it does argues for little intermixing of parental genomes over large tracks of the genome. However, there has been considerable change at the 18-5.8-26S rDNA loci.

In $N$. paniculata, which has two 18-5.8-26S rDNA loci, there is a uniform length of the basic 18-5.8-26S rDNA subunit, and restriction analysis indicates one predominant IGS type (Figures 3 and 5). In N. undulata, which has three 18-5.8-26S rDNA loci, there is also a uniform length of the 18-5.8-26S rDNA subunit, and only a few polymorphisms in the IGS are revealed with some enzymes (eg Bst NI) indicating several (three or four) similar subunit types (Figures $3 \mathrm{~d}$ and 5). In contrast, $N$. rustica has at least three classes of rDNA unit length over the five rDNA loci and considerable heterogeneity of IGS at the $5^{\prime}$ end of the sequence (Figures 3 and 5). In the majority $(80 \%)$ of these units, there is high similarity at the $3^{\prime}$ end of the IGS to sequences in N. undulata.

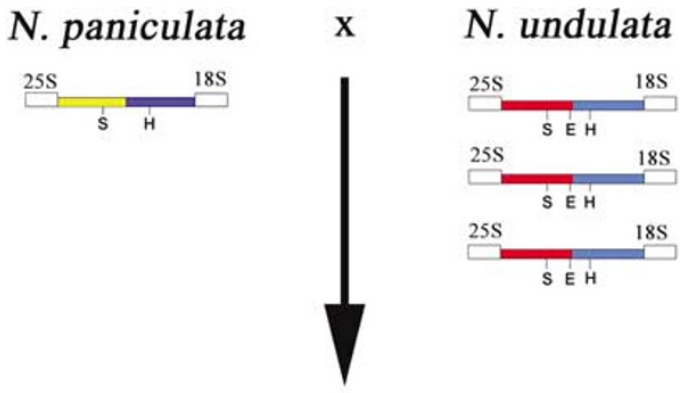

\section{N. rustica}

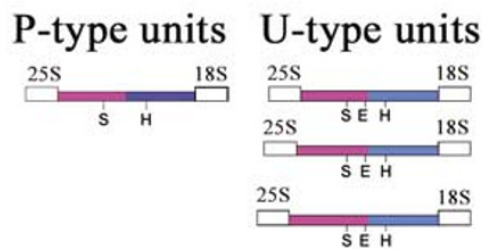

Figure 5 Schematic diagram showing the IGS structure between the $26 \mathrm{~S}$ and $18 \mathrm{~S}$ rDNA subunits of $N$. paniculata, N. undulata and $N$. rustica. The colour coding gives an indication of similarity of units. The relative positions of EcoRV (E), HindIII (H) and SspI (S) target sites are shown in rDNA unit variants.
The restriction patterns revealed with the $18 \mathrm{~S}$ rDNA probe indicate sequence homogenisation across many rDNA units in N. rustica. The N. paniculata-type subunits are under-represented in N. rustica, and this may have arisen via recombination between $\mathrm{P}$ - and U-genome units within the IGS followed by homogenisation, so that now only $\mathrm{ca} 20 \%$ of the units have an N. paniculata-like character. It is possible that this process happened early in the divergence of $N$. rustica, and it may have been associated with allopolyploidy itself, which can stimulate genome reorganisation in some species (Ozkan et al, 2001). It is also likely that the length of the IGS slowly converges to a uniform size by dynamic compensation of deletions and amplifications of IGS subrepeats (Volkov et al, 1999).

Evidence from plastid DNA sequence data indicates that an ancestor of $N$. paniculata is the maternal genome donor (Aoki and Ito, 2000). Gill (1991) suggested in the nucleocytoplasmic interaction hypothesis that the paternal genome of newly formed allopolyploids was most likely to reorganise to accommodate an alien cytoplasmic background. However in N. rustica, the units of rDNA from $N$. paniculata, the putative maternal genome, seem to have undergone the greatest change. Thus, as in tobacco (Volkov et al, 1999; Lim et al, 2000b), interlocus gene conversion has occurred so that the IGS of the paternal genome donor is predominating. Furthermore, combined GISH and rDNA in situ hybridisation revealed that the largest, most active rDNA loci are on the U-genome chromosomes, indicating expansion of the $N$. undulata-type units at these loci. The small size of the P-genome loci might suggest that this is associated with a reduction in the number of units on the P-genome chromosomes.

The character of the IGS in N. rustica appears different when $26 \mathrm{~S}$ rDNA is used as the probe. The probe detects polymorphisms occurring predominantly at the $5^{\prime}$ end of the IGS, and with most enzymes a unique pattern of bands in $N$. rustica was observed. Only one enzyme (BstNI) revealed an $N$. undulata-like pattern similar to those seen with the 18S rDNA probe. These data indicate either IGS sequence divergence subsequent to homogenisation or a different IGS character in the true parent of $N$. rustica. If sequence divergence is influencing the $5^{\prime}$ end of the IGS more than the $3^{\prime}$ end of the sequence, this probably reflects functional constraints associated with the promoter, regulator and externally transcribed spacer sequences found in the $3^{\prime}$ region.

Homogenisation of rDNA is often, but not always, observed at rDNA loci. In wheat (Triticum aestivum), a 10000-year-old allohexapolyploid, subrepeats are found in the IGS (Barker et al, 1988) as in tobacco (Volkov et al, 1999). In wheat, the structure of the A-type subrepeat varies little, but their copy number differs among loci and cultivars (Flavell, 1990). In wild populations of tetraploid wheats, the IGS in some populations is heterogeneous whereas in others it is homogeneous (Flavell et al, 1986), indicating both divergence, homogenisation and fixation of subrepeat number (Flavell, 1989), processes that are probably similar to what has occurred in N. rustica. In Gossypium there has also been interlocus concerted evolution of rDNA units in several allopolyploid species, and homogenisation has occurred bidirectionally in allopolyploid species with similar genetic backgrounds (Wendel et al, 1995b). However 
gene conversion appears not to have homogenised loci in Brassica allotetraploids (Chen and Pikaard, 1997).

Lim et al (2000b) argued that a major controlling factor for the occurrence and extent of sequence homogenisation by gene conversion was rRNA gene activity at interphase when the DNA is most decondensed. It is possible that sequence homogenisation occurs when activity at interphase is coupled with mutual homology between IGSs of different rDNA units. The direction of change may be random, except that the most abundant sequence type would most likely be fixed. If this reasoning is correct and there is high mutual homology between IGS sequences, then those variants associated with active, expressed rDNA units at interphase are likely to predominate. Cytosine methylation of rDNA units was higher in N. rustica compared with the diploid progenitor species, particularly at the minor fraction of units that have not been homogenised and retained the N. paniculata-like character (see XhoI and Fnu4HI digests, Figure 4). Similarly, in N. tabacum the minority N. sylvestris-type units are heavily methylated (Lim et al, $2000 \mathrm{~b})$. Heavily methylated units are not usually active at interphase; indeed inactive rDNA loci can be experimentally activated by the addition of demethylating agents (Vieira et al, 1990; Chen and Pikaard, 1997). If Lim et al (2000b) are correct, then it can be predicted that there is lower activity of the rDNA loci on the P-genome chromosomes of $N$. rustica and this is a factor contributing to the maintenance of some $N$. paniculata-like units in the allotetraploid. While there is some evidence of reduced 18-5.8-26S rDNA activity in the P-genome chromosomes of root-tip interphases, more direct proof that this is inhibiting interlocus homogenisation requires the analysis of rDNA condensation in undistorted nuclei, from germ-line tissue of resynthesised synthetic N. rustica, with the parental origin of the rDNA units and loci identified by specific in situ markers.

\section{Acknowledgements}

We thank NERC and the Grant Agency of the Czech Republic (Grants 204/01/0313 and 521/01/0037) for funding. We thank Miss D Saikia and Mr J Clarkson for assistance, and Professor M Chase and Dr I Leitch for helpful comments.

\section{References}

Aoki S, Ito M (2000). Molecular phylogeny of Nicotiana (Solanaceae) based on the nucleotide sequence of the matK Gene. Plant Biol 2: 316-324.

Barker RF, Harberd NP, Jarvis MG, Flavell RB (1988). Structure and evolution of the intergenic region in a ribosomal DNA repeat unit of wheat. J Mol Biol 201: 1-17.

Chase MW, Cox AV, Clarkson J, Knapp S, Marshall JA, Parokonny AS (2003). Molecular systematics, GISH and the origin of hybrid taxa in Nicotiana. Ann Bot 92: 107-127.

Chen ZJ, Pikaard CS (1997). Epigenetic silencing of RNA polymerase I transcription: a role for DNA methylation and histone modification in nucleolar dominance. Genes Dev 11: 2124-2136.

Flavell RB (1989). Variation in structure and expression of ribosomal DNA loci in wheat. Genome 31: 963-968.
Flavell RB (1990). The molecular basis of variation affecting gene expression: evidence from studies on the ribosomal RNA gene loci of wheat. In: Gustafson JP (ed) Gene Manipulation and Improvement, Plenum Press: New York. Vol II, pp 419-429.

Flavell RB, Odell M, Sharp P, Nevo E, Beiles A (1986). Variation in the intergenic spacer of ribosomal DNA of wild wheat, Triticum dicoccoides, in Israel. Mol Biol Evol 3: 547-558.

Fulnecek J, Lim KY, Leitch AR, Kovarik A, Matyasek R (2002). Evolution and structure of 5S rDNA loci in allotetraploid Nicotiana tabacum and its putative parental species. Heredity 88: 19-25.

Gerlach WL, Bedbrook JR (1979). Cloning and characterization of ribosomal RNA genes from wheat and barley. Nucleic Acids Res 7: 1869-1885.

Gill BS (1991). Nucleocytoplasmic interaction (NCI) hypothesis of genome evolution and speciation in polyploid plants. In: Sasakuma T, Kinoshita T (eds) Proceedings of the Kihara Memorial International Symposium on Cytoplasmic Engineering in Wheat, Yokohama, Japan, pp 48-53.

Gill BS, Friebe B (1998). Plant cytogenetics at the dawn of the 21st century. Curr Opi Plant Biol 1: 109-115.

Goodspeed TH (1954). The Genus Nicotiana. Chronica Botanica Company: MA, USA.

Heslop-Harrison JS (1992). Molecular cytogenetics, cytology and genomic comparisons in the Triticeae. Hereditas 116: 93-99.

Kiss T, Kiss M, Solomosy F (1989a). Nucleotide sequence of $25 \mathrm{~S}$ rDNA gene from tomato. Nucleic Acids Res 17: 796-800.

Kiss T, Szkulek A, Solomosy F (1989b). Nucleotide sequence of a $17 S$ (18S) rDNA gene from tomato. Nucleic Acids Res 17: 21-27.

Kovarik A, Fajkus J, Koukalova B, Bezdek M (1996). Speciesspecific evolution of telomeric and rDNA repeats in the tobacco composite genome. Theor Appl Genet 92: 1108-1111.

Leitch IJ, Bennett MD (1997). Polyploidy in angiosperms. Trends Plant Sci 2: 470-476.

Leitch AR, Lim KY, Webb DR, McFadden GI (2001). In situ hybridisation. In: Hawes C, Satiat-Jeunemaitre B (eds) Plant Cell Biology, a Practical Approach, Oxford University Press: Oxford.

Leitch AR, Schwarzacher T, Jackson D, Leitch IJ (1994). In situ Hybridisation: a Practical Approach. Bios Scientific Publisher: Oxford.

Lim KY, Kovarik A, Matyasek R, Bezdek M, Lichtenstein CP, Leitch AR (2000b). Gene conversion of ribosomal DNA in Nicotiana tabacum is associated with undermethylated, decondensed and probably active gene units. Chromosoma 109: 161-172.

Lim KY, Leitch IJ, Leitch AR (1998). Genomic characterisation and the detection of raspberry chromatin in polyploid Rubus. Theor Appl Genet 97: 1027-1033.

Lim KY, Matyasek R, Lichtenstein CP, Leitch AR (2000a). Molecular cytogenetic analyses and phylogenetic studies in the Nicotiana section Tomentosae. Chromosoma 109: 245-258.

Murad L, Lim KY, Christopodulou V, Matyasek R, Lichtenstein CP, Kovarik A, Leitch AR (2002). The origin of the paternal genome of tobacco is traced to a particular lineage within Nicotiana tomentosiformis (Solanaceae). Am J Bot 89: 921-928.

Olmstead R, Palmer JD (1991). Chloroplast DNA and systematics in the Solanaceae. In: Hawkes JG, Lester RN, Nee M, Estrada N (eds) Solanaceae III. Taxonomy, Chemistry and Evolution. Kew, Royal Botanic Gardens: Kew, UK. pp 161-168.

Ozkan H, Levy AA, Feldman M (2001). Allopolyploidy-induced rapid genome evolution in the wheat (Aegilops-Triticum) group. Plant Cell 13: 1735-1747.

Parokonny AS, Kenton AY (1995). Comparative physical mapping and evolution of the Nicotiana tabacum L. karyotype. In: Brandham PE, Bennett MD (eds) Kew Chromosome Conference II, Royal Botanic Gardens: Kew, UK. pp 301-320. 
Saghai-Maroof MA, Soliman KM, Jorgensen RA, Allard RW (1984). Ribosomal DNA spacer-length polymorphisms in barley - Mendelian inheritance, chromosomal location, and population-dynamics. Proc Natl Acad Sci USA 81: 8014-8018.

Sambrook J, Fritsch EF, Maniatis T (1989). Molecular Cloning: a Laboratory Manual. Cold Spring Harbor Laboratory Press: New York, USA.

Soltis DE, Soltis PS (1999). Polyploidy: recurrent formation and genome evolution. Trends Ecol Evol 14: 348-352.

Venkateswarlu K, Lee S-W, Nazar RN (1991). Conserved upstream sequence elements in plant $5 \mathrm{~S}$ ribosomal RNAencoding genes. Gene 105: 249-253.

Vieira R, Queiroz A, Morais L, Barao A, Mellosampayo T, Viegas W (1990). Genetic-control of 1R nucleolus organizer region expression in the presence of wheat genomes. Genome 33: $713-718$.
Volkov RA, Borisjuk NV, Panchuk II, Schweizer D, Hembeleben V (1999). Elimination and rearrangement of parental rDNA in the allotetraploid Nicotiana tabacum. Mol Biol Evol 16: 311-320.

Volkov RA, Zanke C, Panchuk II, Hemleben V (2001). Molecular evolution of $5 \mathrm{~S}$ rDNA of Solanum species (sect. Petota): application for molecular phylogeny and breeding. Theor Appl Genet 103: 1273-1282.

Wendel JF, Schnabel A, Seelanan T (1995a). An unusual ribosomal DNA-sequence from Gossypium gossypioides reveals ancient, cryptic, intergenomic introgression. Mol Phylogenet Evol 4: 298-313.

Wendel JF, Schnabel A, Seelanan T (1995b). Bidirectional interlocus concerted evolution following allopolyploid speciation in cotton (Gossypium). Proc Natl Acad Sci USA 92 280-284. 